\title{
Holonic Manufacturing Systems
}

\author{
M. Höpf, C.F. Schaeffer \\ Fraunhofer Institute \\ Manufacturing Engineering and Automation \\ Nobelstrasse 1270569 Stuttgart, Germany \\ Fax: $+49(0) 711970-1008$ \\ Phone: $+49(0) 711970-1127$ \\ e-mail: hoepf@ipa.fhg.de
}

\begin{abstract}
This contribution introduces in the background of holonic systems and holarchies as a hierachical model for biological and social structures in nature. The characteristics of these hierarchical architectures and its elements will be explained. It will be shown how this approach can be used as a potential solution for present and future challenges in manufacturing control systems. The results of an internationally performed feasability study heading for the joint development of this technology will be described. A report on the current status of the international research activities will cover the technical and organisational aspects of the project.
\end{abstract}

\section{Keywords}

Holon, manufacturing, IMS, control system, architecture. 


\section{THE BACKGROUND OF HOLONIC ARCHITECTURES}

\section{The History of Holarchies}

The expressions „holon“ and „holarchy“ first appeared in 1967 in Arthur Koestlers book „The ghost in the machine“. Koestler was a bulgarian born journalist and spend most of his life with biological and social studies to explain the human fate. "The ghost in the machine" is the last book of a trilogy where he analyses biological and social evolution to proof that the historical behavioristic approach with its mechanistic world-view is not an appropriate model for the organisation of living structures. It was very important for him to show that human beeings are not a kind of biological robots whose behaviour is predetermined by their role in fixed hierarchies. Instaed of characterizing a system as a whole and the elements as dependent parts of it, Koestler found out that the functional properties of social phenomena as well as biological sructures require that the elements have tendency to integrate themselvs in a structure but at the same time try to preserve their autonomy as self contained independent units.

Arthur Koestlers motivation for this kind of research was his own fate. Born in 1905 he past most of his life in Europe,Palestine and Russia as a journalist and writer of novels and essays. His engagement with communism and the fact that he was a jew was the reason that he was put under pressure from various authorities and he even had to pass several years in the jail in spain. There he started to research the structures of biological life by analyzing the interaction of organs in animals and the human body. He compared the structures he found with social systems and developed the open hierarchical systems (OHS) as a general organisation principle of life.

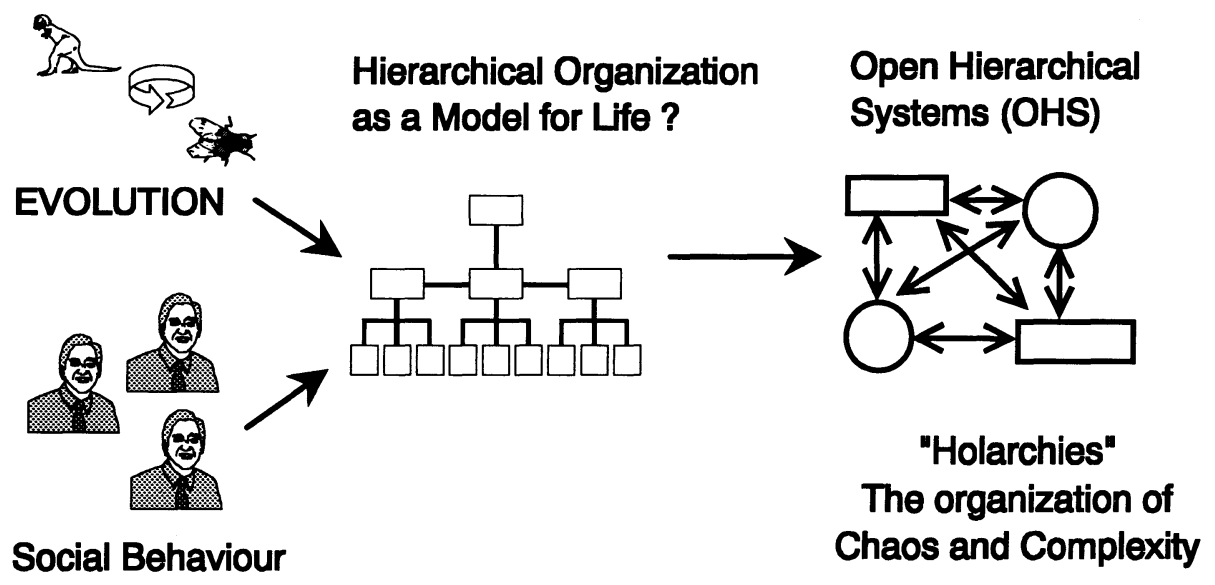

Figure 1 Architecture of natural systems. 


\section{The System "life“}

The model Koestler developed for the system "life“ is based on the fact that hierarchies are an essential element in living structures but these hierarchies are not fixed. Living organisms obviously can change their structures and are dissectable. This enables the systems to use inbuild deficencies and defects to optimise their function according to present objectives as for example survival in dangerous situations. Hierarchies in nature are therefore temporary. This is more obvious in social systems where the ability of self-adapting hierarchies is a basic principle of democracy. Fixed subordination as functional element does not exist in nature (biological and social structures). The organisation of living systems is characterized by a constantly changing network where the elements play the role of exchangeable nodes and the links are represented as interactions like communication, negotiation and even agressions. These interactions can be based on external or internal events and allways result in a change in the temporary hierarchical structure and subordination scheme.

Evolution and social development are the logical consequence of this self-organising systems, where even the top of hierarchies are not fixed. Systems which do not have these properties are not stable in nature. Evolution and survival depend on a constant transition in structures. This requires, that all elements of the system are not designed to take over a predetermined role in a hierarchy but are autonomous and self-similar. Living systems cannot be divided in the whole and parts of it or in components and the system. Elements that have this autonomy are called „Holons“ and their behaviour is described by Koestler in a set of rules. The architecture of systems which are composed of Holons is called „Holarchy“. Living systems are either unstable or holarchies.

\section{Holonic Systems Characteristics}

One of the most important characteristics of Holons is the Janus-Effect. While the Holons display autonomy they tend also to integrate themselfs into a hierarchy. If Holons are given the possibility of interaction and rules for this interaction they will automatically form a system and attract further holons for integration. Thus holonic systems are self-developing and have the inherent tendency to grow. Since the holons are autonomous the systems are dissectable. Parts of the systems or individual holons may be separated or organised in different holarchies. The holons try to maintain a dynamic equilibrum between their integrative and autonomous tendencies. If this equilibrum is disturbed, the system tries to reorganize itself. The goal of this reorganisation is the adoption of the network to changed conditions and the optimized function of the system. This process is initiated by the holons tendency to cooperate. 


\title{
Basic Properties of Holarchies
}

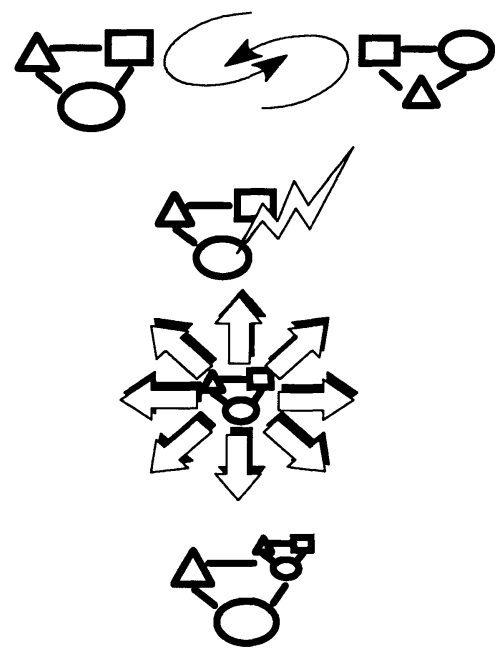

\author{
Dynamic Structure
}

Stability and Robustness

Sustainable Growth

Self-Similarity

Figure 2 Basic properties of holarchies.

\section{HOLONIC SYSTEMS IN MANUFACTURING TECHNOLOGY}

\section{New Challenges in Production}

Industrial manufacturing is at the beginning of a technical revolution. Fast innovation cycles, complex products and decreasing product life cycles are the new challenges in competitive global manufacturing. Manufacturing is changing more and more to a self-contained business which has to be effective and less expensive than today. The fast development of new products and processes becomes a problem for large series lines and fixed production installations. The costs for installation changes and reconfiguration in a line reach one third of overall costs. The reliability of hierarchical organized manufacturing systems becomes uncontrollable if the systems get too complex.

New enterprise organisation schemes like the fractal factory are already in course of implementation as a reaction on these environmental changes.

On the technical level of production control hierarchical structures are still standard. Decentralized architectures are currently under development but still their integration effort is too high to be competitive. Moreover any change in the system is has to be regarded as a disturbance or even as a breakdown, but today the number of changes caused by the environment requires a constant transition of a line during operation.

The current level of automation can only be maintained if these problems are solved and the control systems of manufacturing systems support constant and on-line change and 
configuration with reduced manual effort. The objective is to lower the costs for electrical installations and dedicated software developments significantly.

Holonic Manufacturing Systems as the result of configuring the controls of individual manufacturing equipment as holons is the key technology to reach this objectives.

\section{Industrial Benefits}

\section{- reduce IT investments in manufacturing lines}

- reuse control equipment and software

\section{- maximize fault tolerance in complex manufacturing systems}
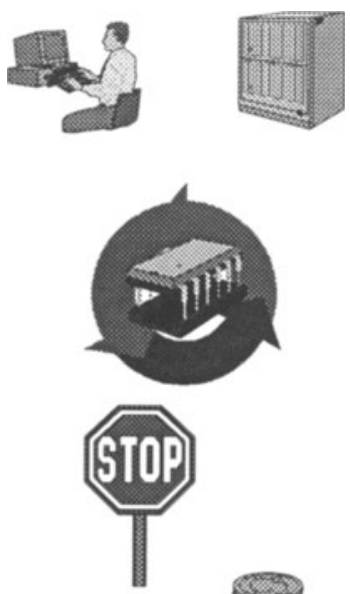

\section{- reduce the cost of changes}

Figure 3 Industrial benefits of industrial controls.

\section{CIM and Holonic Systems}

The development of computer integrated manufacturing has shown that automation has a similar potential for effectiveness in production as the use of computers in business administration. CIM has developed from single machine control and programmable logic controllers to overall production control systems based on information networks. New developments are aiming at open systems and standardized communication channels with the possibility to transfer and process all kind of information on the shop floor. The controls of the process equipment have access to all informations in real time and are able to communicate not only with a central system but with each other. At the same time the capabilities for decentralized complex data processing improve dramatically and the individual control system can be configured as autonomous systems at a reasonable price. What lacks is a common concept to use this functionalities to overcome the problems encountered. In that view holonic manufacturing systems is an implementation strategy for the further developments of CIM. HMS can be considered as a software technology on the top level of the communication 
infrastructure in networked manufacturing machines enabling the use of large information resources for decentralized decision making.

The use of extended information technology in machine controls in conjunction with information about the current production and business strategies results in a self configuration behaviour avoiding extensive efforts for programming and manual definitions of actions and reactions. This can be compared with the self configuration capabilities of computer ad-ons replacing the manual configuration tasks of the respective electronic boards. It is estimated that this functionality will save up to $30 \%$ of the installation costs for a new manufacturing line.

By adding on-line information about the current status of individual machines and the production line to this network, the reliability and availability of complex manufacturing systems is enhanced. In case of a defect or even unforeseen disturbances in normal operation of a line, the analyzing and negotiation capability of the controls is used to investigate auxiliary options in the process and to configure optimal alternatives to maintain the production process.

Due to this qualities holonic control systems are the basis for the effective use of advanced computer integrated manufacturing and industrial communication technolgies.

\section{Technical Background}

\section{self-configurating network \\ of autonomous and cooperative}

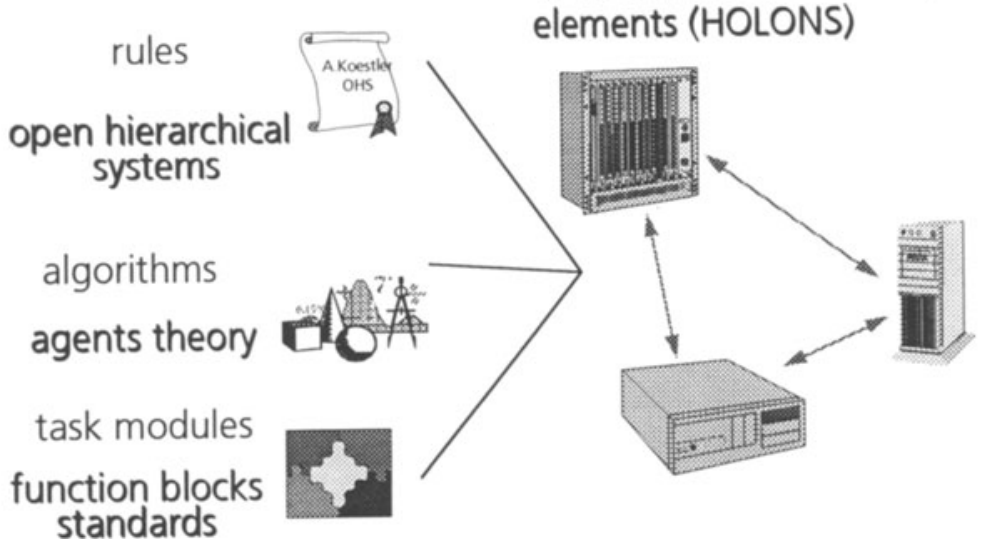

Figure 4 Implementation of holonic control systems.

\section{Development aspects of. Holonic Manufacturing Systems}

Holonic control systems as the basic technology for holonic manufacturing systems will be based on a number of innovative technical approaches which are currently under development. 
One of the most important technical components for a holonic systems is the communication architecture. The field bus systems today available have to be evaluated for their qualification in co-operative networks. The standardisation of the functional architecture is already an ongoing activity.

Holonic Manufacturing systems are the logical consequence of these emerging technologies. With the availability of the communication networks the question what to do with it arises. Just transferring programs and commands or supervising machinery and systems would not justify the expensive equipment and software. Even the co-ordination of complex schedules does not require extensive communication. In this light, HMS adds a new type of functionality to the individual manufacturing component by using the communication for autonomous interaction. This concept is based on the scientific theory of agent systems. This theory provides the algorithms for negotiation and decision making in artificial intelligence. HMS adds the rules and common contracts to such a system in the context of a dedicated manufacturing system.

\section{THE INTERNATIONAL HMS PROJECT}

In 1993 a feasibility study was commenced to evaluate the possibilities of international cooperation for the development of HMS. This study was one of six test-cases launched under the international IMS programme and was carried out by a consortium of 31 partners from North-America, European Union, Australia and Japan. The study was focused on the investigation of the industrial potential of HMS and the outline of a workprogramme for a joint development project. Starting with the research on user requirements and forecasts for future trends in manufacturing the consortium also developed three testbeds to evaluate the characteristics of HMS in real applications. The results of the feasibility study encouraged the 15 industrial participants to agree on a joint project to develop, produce and market a new type of industrial controllers in a world-wide co-operation.

\section{Project Overview}

Generic Workpackages:

$$
\begin{aligned}
& \text { Architecture \& Engineering Systems Operation } \\
& \begin{array}{ll}
\frac{\text { Application }}{\text { Workpackages }} & \text { - Resource Management } \\
\text { - Manufacturing Units } & \text { - Fixturing } \\
\text { Holonic } & \text { - Mandling } \\
\end{array}
\end{aligned}
$$

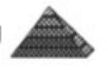$$
\text { (3) }
$$

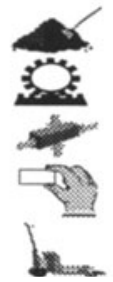

Figure 5 The HMS Project. 
In the following interim phase the consortium was restructured and a contract framework was developed. On the basis of the Terms of Reference as an international background a consortium co-operation agreement was negotiated. This agreement basically regulates the creation and use of intellectual property within the consortium. A set of workpackage agreements include the workprogrammes and the contributions of the consortium partners.

The workprogramme comprises two generic workpackages and five application workpackages. The generic workpackages will develop the design technology and the basic function in close co-operation with current standardisation activities. The application workpackages are will implement these concepts in primary industrial areas identified in the feasibility study. Extensive technology interchange mechanisms will ensure that application experiences are evaluated in parallel by the generic workpackages and the basic design and architecture will be compatible in the applications.

The contracts and the workprogramme have been accepted by the international steering committee of the IMS Programme in late 1995. The consortium is now preparing the start of the development project.

\section{BIOGRAPHIES}

\section{Christoph F. Schaeffer}

Research Scientist, Group Leader, Motion Control Systems and Sensor Guidance, Dipl.-Ing., Electrical Engineering/Computer Science, RWTH Aachen, 1986.

Project Leader for prototype developments in mobile and service robotics, since 1987.

Proposer and Project Manager of European Research Project ESPRIT 5292 MOSAIC modular motion control system with unified interfaces, 1990-1992.

Co-proposer and International Working Group Leader of the Transport Group within the IMS test case project Holonic Control Systems (HMS), 1993/94.

Member of DIN/NAM working group to ISO/WD 11513.1 (programming languages).

Special personal expertise in control system architectures, production planning and scheduling.

Michael Höpf was born in 1955 in Saarbrücken, Germany. In 1983 he concluded his study in technical and economical Cybernetics in Stuttgart as Diplom-Ingenieur. In the same year he started working as a junior scientist at the Fraunhofer Institute for Manufacturing Technology and Automation, IPA. His working area was robotics application in machining. In 1988 he became group leader in the department of sensor technology and Robot Systems at the IPA. There he was responsible for various industrial contract developments in the area of sensor systems and industrial software.

He was also active in public founded consultancy projects to support SMEs in implementing new manufacturing technologies. First experiences with the initiation and the management of EU-founded projects where gained in Brite-Euram and ESPRIT Projects where the institute took over the role as a co-ordinator.

Since 1993 he participates in the HMS Project where he became official delegate in the management committee in 1994. 